\title{
What Evidence Is There That Gadobutrol Causes Increasing Signal Intensity within the Dentate Nucleus and Globus Pallidus on Unenhanced T1W MRI in Patients with RRMS?
}

\author{
Jacob Agris $^{1} \cdot$ Hubertus Pietsch $^{2} \cdot$ Thomas Balzer $^{1}$
}

Received: 28 August 2015 / Accepted: 7 September 2015 / Published online: 21 September 2015

(C) The Author(s) 2015. This article is published with open access at Springerlink.com

Key Points

- The study by Stojanov et al does not convincingly support the conclusion that gadobutrol causes higher T1 enhancement in brain on unenhanced MRI

- The study by Stojanov et al does not rule out confounding factors

- The study by Stojanov et al has limitations in study design

Keywords Gadobutrol · Signal intensity on unenhanced MRI · Dentate nucleus · Globus pallidus

We read with great interest the recent article by Stojanov et al. [1]. In this article, the authors state that "... an increase in SI within the dentate nucleus and globus pallidus on unenhanced $T 1 W$ images in patients with RRMS may be a consequence of multiple administrations of gadobutrol. Administration of the same amount of gadobutrol over a shorter time period causes a greater increase in SI within the dentate nucleus and globus pallidus."

The authors' findings are new as this is the first time that a macrocyclic gadolinium-based contrast agent (GBCA) is suggested to be associated with this phenomenon. Prior studies, such as the 2009 Roccatagliata et al study [2], where the patients received gadoterate meglumine (Dotarem) and gadodiamide (Omniscan), reported hyperintensity in dentate nuclei in patients with secondary progressive MS, which they

Thomas Balzer

thomas.balzer@bayer.com

1 Global Medical \& Clinical Affairs Radiology, Bayer HealthCare, 100 Bayer Boulevard, Whippany, NJ 07981, USA

2 MR \& CT Contrast Media Research, Bayer HealthCare, Whippany, NJ, USA attributed to, among other things, the disease and particularly the severity of the disease process itself, or potentially linked to the associated iron depositions as demonstrated in tissue specimens by Craelius et al. [3].

The title and abstract suggest an association between high signal intensity (SI) and a macrocyclic GBCA, which is less likely to release free gadolinium. However, the study design and authors' data do not rule out a number of potentially confounding factors that could contribute to any direct association, though some of the limitations have been acknowledged in the author's discussion. For example:

- Although other causes or the disease process itself has been proposed as causes for the hyperintensity, this retrospective comparison did not include a control group of patients with no CNS or a non-MS disease.

- The authors specifically acknowledge that they could not control for or exclude the use of other contrast agents, including linear GBCAs. The fact that the baseline values (before the first gadobutrol dose) show elevated SI raises the possibility that there may have been either confounding contrast agent contamination prior to the first contrastenhanced MRI that was used as the reference or other causes such as the previously discussed disease itself or associated iron depositions, which may play a critical role [3].

- Patients were evaluated in three groups with different intervals of contrast administration. The different intervals between contrast-enhanced MRI may be indicative of different disease activity and/or treatment regimens requiring more frequent follow-up exams. This could introduce a bias if the disease activity itself is contributing to the hyperintensity. This might explain why the most prominent effect was in the group with the shortest period of contrast administration.

- The scatter plot (Fig. 3) shows a correlation of 0.263, which is very low when compared to other similar studies, 
suggesting a potential unknown cause other than the administration of gadobutrol [4]. As common practice, a correlation of 0.50 or more is regarded as moderate and correlations of 0.75 or above as strong. Additionally, the number of administrations showed a statistically significant correlation to the increase in SI, but the total amount of contrast did not. This creates unanswered questions because the number of administrations and total amount of contrast are highly correlated.

- Any visible changes in SI are not specifically mentioned and are difficult to appreciate even on the selected images provided in the paper, but only SI measurements are reported. Prior publications on MS have explored the importance of the region size and location on the quantitative measures, but this study does not specify any control to ensure the regions on the first and last scans were similar in size and location. The regions-of-interest may have been determined anatomically by consensus, rather than based on observable areas of signal increase.

In summary, there are concerns that limitations in the study design do not support any conclusion regarding the role of repeated administrations of gadolinium-based contrast agents in general or a specific contrast agent in this particular patient group.
Open Access This article is distributed under the terms of the Creative Commons Attribution-NonCommercial 4.0 International License (http://creativecommons.org/licenses/by-nc/4.0/), which permits any noncommercial use, distribution, and reproduction in any medium, provided you give appropriate credit to the original author(s) and the source, provide a link to the Creative Commons license, and indicate if changes were made.

\section{References}

1. Stojanov DA, Aracki-Trenkic A, Vojinovic S, BenedetoStojanov D, Ljubisavljevic S. Increasing signal intensity within the dentate nucleus and globus pallidus on unenhanced T1W magnetic resonance images in patients with relapsingremitting multiple sclerosis: correlation with cumulative dose of a macrocyclic gadolinium-based contrast agent, gadobutrol. Eur Radiol. 2015 Jun 25.

2. Roccatagliata L, Vuolo L, Bonzano L, Pichiecchio A, Mancardi GL (2009) Multiple sclerosis: hyperintense dentate nucleus on unenhanced T1-weighted MR images is associated with the secondary progressive subtype. Radiology 251(2):503-10

3. Craelius W, Migdal MW, Luessenhop CP, Sugar A, Mihalakis I (1982) Iron deposits surrounding multiple sclerosis plaques. Arch Pathol Lab Med 106(8):397-9

4. McDonald RJ, McDonald JS, Kallmes DF, Jentoft ME, Murray DL, Thielen KR et al (2015) Intracranial Gadolinium Deposition after Contrast-enhanced MR Imaging. Radiology 275(3):772-82 